\title{
Evidencia y uso actual de toxina botulínica en patología otorrinolaringológica
}

\section{Evidence and actual use of botulinum toxin in otolaryngology}

Jorge Caro $L^{1}$, Nicolás Fuentes $L^{2}$, Rodrigo Iñiguez $C^{1}$.

\begin{abstract}
RESUMEN
A partir de los primeros usos terapéuticos de la toxina botulínica su utilización se ha extendido a variadas ramas de la medicina incluyendo la otorrinolaringología. Se considera un medicamento seguro en manos de profesionales capacitados y su utilización se realiza a través de un procedimiento mínimamente invasivo con efectos adversos leves y transitorios. Se usa en un amplio número de patologías otorrinolaringológicas y de cabeza y cuello.

Actualmente la toxina botulínica $A$ representa la primera línea de tratamiento en pacientes con disfonía espasmódica y distonía cervical. Existe evidencia de buen nivel que recomienda su uso para el manejo de sialorrea, temblor laríngeo, trastornos de la ATM, bruxismo y rinitis en casos seleccionados. Presenta buenos resultados en otras patologías como espasmo faringoesofágico, síndrome de Frey y tinnitus, sin embargo, todavía son necesarios más estudios.
\end{abstract}

Palabras clave: Toxina botulínica, botox, otorrinolaringología.

\section{ABSTRACT}

Since the first therapeutic uses of botulinum toxin its role has extended to a wide range of medicine áreas including otolaryngology. It is considered a safe medication in the hands of trained professionals and its application represents a minimally invasive procedure with mild and transient adverse effects. It has been used in a wide range of otolaryngology and head and neck pathologies.

Today botulinum toxin a represents the first choice of treatment for patients with spasmodic dysphonia and cervical dystonia. There is good evidence that supports its use for shialorrea, laryngeal tremor, temporomandibular joint disorders, bruxism and selected cases of rhinitis. For pharyngo-oesophageal spasm, Frey's syndrome and tinnitus there have been studies that show a beneficial effect however more studies are required for routine recommendation.

Key words: Botulinum toxin, botox, otolaryngology.

\footnotetext{
${ }^{1}$ Médico Servicio de Otorrinolaringología, Hospital Clínico de la Pontificia Universidad Católica de Chile.

${ }^{2}$ Interno de Medicina, Hospital Clínico de la Pontificia Universidad Católica de Chile.
} 


\section{INTRODUCCIÓN}

La toxina botulínica es producida por la bacteria Clostridium botulinum y se considera uno de los venenos más potentes que existen ${ }^{1}$. La intoxicación se manifiesta a través del botulismo, cuadro clínico que se caracteriza por debilidad muscular generalizada y posterior falla respiratoria secundarias a la ingestión de salsas con carne contaminada.

Los primeros casos de botulismo fueron reportados en 1820 y posteriormente en 1895 Van Ermengem y Kempner lograron aislar el bacilo anaerobio ${ }^{2}$. En Chile los primeros casos confirmados por el Instituto de Salud Pública ocurrieron en la década de los 70 a raíz de la ingestión de legumbres contaminadas en un convento ${ }^{3}$. A partir de 1943 se comenzó a trabajar para purificar la toxina. Carl Lamanna logra aislar la toxina botulínica tipo A y a partir de 1972 se inician sus primeras aplicaciones terapéuticas para controlar el estrabismo. Finalmente se extiende su uso a otras patologías como el blefaroespasmo.

Se ha aprobado su uso por la FDA (Food and Drug Administration) con fines terapéuticos desde 1989. Actualmente, su uso más conocido es el estético con más de 20 años de experiencia y excelentes resultados sobre todo en tratamiento del tercio superior de la cara ${ }^{4}$ dada la menor incidencia de asimetría y efectos adversos.

\section{FARMACOLOGÍA}

Existen 8 serotipos distintos de toxina botulínica (AC1 y C2-G). Todos son proteasas que actúan a nivel de la placa neuromuscular evitando la liberación de acetilcolina ${ }^{5}$. Los serotipos A y B son los que tienen aplicación clínica. El serotipo B no está disponible en Chile.

El más utilizado es la toxina botulínica tipoA (TbA) que actúa sobre la proteína SNAP-25 generando una denervación química temporal que se recupera en dos fases. Primero se generan terminales accesorios y posteriormente se produce la recuperación del axón afectado alrededor de 90 días luego de la aplicación ${ }^{6}$.

Actualmente en Chile están disponibles dos marcas de TbA purificada. Ellas son Botox ${ }^{\circledR}$ de
Allergan (USA) y Dysport $^{\circledR}$ de Speywood Pharmaceuticals (UK). Ambas presentaciones traen la toxina en seco por lo que deben reconstituirse con suero fisiológico sin preservantes. La dosis se mide en Unidades Internacionales (UI) que se refiere a la dosis letal media intraperitoneal inyectada en ratones Swiss-Webster. A pesar de esto, 1 UI de Botox ${ }^{\circledast}$ no es equivalente a $1 \mathrm{UI}$ de Dysport ${ }^{\circledast}$ por lo que las dosis no son intercambiables entre sí y tampoco se recomienda transformar una dosis según equivalencia entre las marcas.

Vienen en envases de 100 y $500 \mathrm{UI}$ de Botox ${ }^{\circledR}$ y Dysport $^{\circledR}$, respectivamente.

\section{CONTRAINDICACIONES Y TOXICIDAD GENERAL}

Las contraindicaciones absolutas para el uso de toxina botulínica son infección en el sitio de inyección (incluyendo infección urinaria en inyecciones en el detrusor) y alergia a alguno de sus componentes. Hay que tener especial cuidado en grupos de pacientes que tienen riesgos de presentar complicaciones como aquellos con enfermedades neuromusculares (esclerosis lateral amiotrófica, miastenia gravis, síndrome de Lambert-Eaton, entre otros). También en aquellos con problemas respiratorios y en pacientes que estén en tratamiento con aminoglicósidos o alguna droga que interfiera en la unión neuromuscular ya que se ha visto que pueden presentar profundización del efecto. En pacientes embarazadas sólo debe ser usado si el beneficio supera los riesgos dado que es categoría $C$ ya que en algunos estudios en animales se ha observado que produce retardo en el crecimiento fetal?.

Con respecto a la toxicidad de la toxina botulínica purificada, si bien no existen estudios en humanos, se ha estimado una dosis letal de $40 \mathrm{UI}$ por kilo de peso $02.800 \mathrm{UI}$ en un adulto promedio de 70 kilos. Estos datos fueron extrapolados de un estudio en simios ${ }^{8}$. Es importante destacar que a pesar de que es considerado uno de los venenos más potentes, la cantidad de toxina purificada que viene en los envases de $100 \mathrm{UI}$ de Botox ${ }^{\circledR}$ es de 4,8 nanogramos y la dosis de toxina que es capaz de generar botulismo grave es de $1 \mathrm{mg} / \mathrm{kg}$. Se considera un medicamento seguro en manos entrenadas y no se han reportado muertes debido a su uso terapéutico. 
Existe descrito el fenómeno de resistencia a la TbA principalmente en pacientes que han usado la toxina por largo tiempo y en dosis altas. El mecanismo es a través de la aparición de anticuerpos anti-TbA ${ }^{9}$. No existen estudios que evalúen directamente, luego de cuánto tiempo, ni con qué dosis aparece resistencia, sin embargo se recomienda no administrar más de $300 \mathrm{UI}$ de Botox ${ }^{\circledR}$ en un período de 3 meses.

En Estados Unidos y algunos países de Europa se comercializa la toxina botulínica $\mathrm{B}\left(\mathrm{MyoBloc}^{\circledR}\right)$ que se ha utilizado en casos de resistencia, no está disponible en Chile y presenta mayor incidencia de efectos adversos, sobre todo relacionados al aparato cardiovascular.

\section{USOS ACTUALES}

A lo largo de los últimos años el uso terapéutico de toxina botulínica se ha ampliado a una gran variedad de patologías lo que se ha reflejado también en el ámbito otorrinolaringológico. El presente texto pretende revisar la evidencia actual del uso de toxina botulínica en patología otorrinolaringológica.

\section{1) Patología facial}

Existe evidenciade buenacalidad queconsisteenestudios clínicos randomizados (y una revisión sistemática en el caso de la distonía cervical) que muestran la utilidad de la toxina botulínica en el blefaroespasmo $0^{10,11}$, distonía cervical ${ }^{12}$ y espasmo hemifacial ${ }^{13}$.

También se ha utilizado para el manejo de las complicaciones de la parálisis facial como las sincinesias $^{14}$ y para inducción de ptosis terapéutica del lado afectado ${ }^{15}$.

Tendría un rol en el manejo del dolor en la neuralgia del trigémino en pacientes refractarios a terapia médica como coadyuvante de ésta, sin embargo faltan mayores estudios para recomendar su uso rutinario en estos pacientes ${ }^{16,17}$.

Existe una revisión sistemática que no recomienda su uso en manejo del dolor cervical crónico ${ }^{18}$.

\section{2) Patología de oído}

Se ha utilizado toxina botulínica para el tratamiento de tinnitus por mioclonía palatina ${ }^{19}$ y estapedial ${ }^{20}$ en donde existen reportes de casos para cada patología en que se inyecta por vía oral en paladar blando y a través de Gelfoam intratimpánico respectivamente. Ambos reportes de casos muestran alivio sintomático de los pacientes.

Existe otro estudio ${ }^{21}$ que evaluó la utilidad de la toxina en tinnitus por otras causas. Fue un estudio doble ciego, randomizado y con ramas cruzadas que mostró que la inyección de Botox ${ }^{\circledast}$ subcutáneo en tres puntos alrededor del pabellón auricular mejora significativamente el tinnitus, en el $26 \%$ de los pacientes, sin embargo en algunos casos la mejoría fue tardía. Por lo que no es posible asegurar que sea sólo efecto de la toxina.

En conclusión, se requieren más estudios para definir el rol de la toxina botulínica en tinnitus.

\section{3) Patología laríngea}

En la Tabla 1 se muestran las diversas patologías laríngeas en que se ha utilizado la toxina botulínica.

La técnica de inyección se puede utilizar para la mayoría de las patologías descritas según el músculo que se quiera inyectar. La dosis inicial recomendada varía entre 1 a $2.5 \mathrm{UI}$ de Botox ${ }^{\circledR}$ por músculo en un volumen ideal de $0.1 \mathrm{ml}$ (máximo $0.2 \mathrm{ml}$ ). El uso de anestesia es discutido y en general depende de la experiencia del grupo. Se han descrito varias técnicas para la inyección de toxina botulínica:

Bajo visión directa y administración transoral y a través de membrana cricotiroidea guiado por electromiografía (EMG) o bajo visión por nasofibroscopía. Existe un estudio ${ }^{22}$ que comparó la inyección bajo EMG con la inyección a ciegas y no encontró diferencias significativas entre ambas,

\section{Tabla 1. Toxina botulínica en patología laríngea}

\begin{tabular}{|l|}
\hline Disfonía espasmódica \\
\hline Temblor laríngeo \\
\hline Voz en pacientes laringectomizados \\
\hline Granuloma vocal \\
\hline Movimiento paradojal de cuerda vocal \\
\hline Tics vocales y tartamudez \\
\hline
\end{tabular}


sin embargo, quien realizó las inyecciones a ciegas era un médico entrenado con amplia experiencia en el tema.

La disfonía espasmódica es un trastorno motor central que se manifiesta como una distonía focal de la musculatura laríngea. Tiene una incidencia estimada de 5,9 por cada 100.000 habitantes ${ }^{23}$. Existen dos tipos principales: la variedad aductora que representa la mayoría de los casos y la variedad abductora. La inyección de toxina botulínica en la musculatura intrínseca laríngea representa la primera línea de tratamiento en esta patología. La serie más grande de casos es aquella publicada por Blitzer y cols con 1.300 pacientes $^{24}$. Este estudio publicado el año 2010 utilizó una dosis promedio de 1,8 Ul de Botox ${ }^{\circledR}$ con una duración promedio de 15,1 semanas. El outcome de voz medido de acuerdo a porcentaje de voz normal reportó el $92 \%$ para la disfonía aductora y el $70,3 \%$ para la abductora. Los efectos adversos reportados para la variedad aductora fueron de $25 \%$ de escape vocal y $10 \%$ de disfagia. Se presentaron principalmente dentro de las 2 primeras semanas de inyección y desaparecieron posteriormente.

En el caso de la abductora, el principal efecto adverso es la disnea ( $2 \%$ en la serie de Blitzer) y es por esto que se inyecta un solo músculo cricoaritenoideo posterior por vez.

El temblor laríngeo también corresponde a un trastorno motor central y se manifiesta como voz con pérdida de fluidez, pausas y alteración del tono. Se presenta en pacientes adultos mayores, con otros temblores como enfermedad de Parkinson y temblor esencial. Doce a $30 \%$ de los que tienen temblor esencial presentan temblor laríngeo.

La inyección para esta patología se realiza en el músculo tiroaritenoideo y existe un estudio clínico randomizado ${ }^{25}$ de 13 pacientes que mostró mejoría en una escala de voz de 5 puntos. El estudio mostró una mejoría máxima de hasta 1,7 puntos del basal. Dentro de los efectos adversos reportados el más importante fue escape vocal en el $84 \%$ de los pacientes y disfagia en $23 \%$. Todos, excepto un paciente, presentaron mejoría a la sexta semana de observación. Un estudio prospectivo ${ }^{26}$ y un reporte de caso $^{27}$ mostraron resultados comparables.

Los estudios muestran que la inyección de toxina botulínica en ambas cuerdas mejora la voz en estos pacientes, pero no de manera completa.
Esto debido probablemente a que existe compromiso de musculatura extralaríngea.

En pacientes laringectomizados uno de los mecanismos que les permite fonar es la voz esofágica y la válvula traqueoesofágica. Cuando se produce un espasmo faringoesofágico estos pacientes presentan disfagia y dificultad en la producción de la voz. Tradicionalmente estos pacientes han sido tratados con dilataciones del esfínter faringoesofágico, miotomías y neurectomías.

Existen varios estudios prospectivos ${ }^{28-30}$, el más grande y reciente ${ }^{31}$ incluye 34 pacientes en que se inyectó toxina botulínica $\left(\right.$ Botox $^{\circledR}$ ) bajo EMG a un solo lado del músculo constrictor faríngeo en dosis de $100 \mathrm{UI}$ y dos pacientes con $50 \mathrm{UI}$. Este estudio mostró que, combinado con terapia vocal, los pacientes presentan una mejoría sostenida de la producción de la voz. En este estudio no hubo efectos adversos.

\section{4) Patología autonómica}

El síndrome de Frey se produce por reinervación aberrante de fibras parasimpáticas hacia la piel luego de cirugía en la glándula parótida. Se caracteriza por sudoración, enrojecimiento y pilo erección al comer en el área afectada. El diagnóstico se apoya en la prueba de Minor que además permite delimitar el área afectada.

Existen reportes de casos en que se inyecta el área afectada con 2 a 2,5 UI de Botox por $\mathrm{cm}^{2}$ de piel con alivio sintomático ${ }^{32,33}$. Si bien falta evidencia de mayor calidad es posible plantear el uso de toxina botulínica en estos pacientes.

\section{5) Patología oral y de glándulas salivales}

La toxina botulínica se ha utilizado para el manejo de diversas patologías de las glándulas salivales ${ }^{34}$ que se enlistan en la Tabla 2.

La mayor evidencia se centra en el manejo de la sialorrea que es una patología que afecta de forma significativa la calidad de vida de los pacientes. Puede ser secundaria a deterioro neurológico, Parkinson, tumores de cabeza y cuello 0 ser idiopática.

En el caso del sialocele, fístulas salivales y sialoadenitis crónica la evidencia es en base a reportes de $\operatorname{cas}^{35}{ }^{35}$ y por lo tanto se requieren más 
Tabla 2. Uso de toxina botulínica en patología de glándulas salivales

\begin{tabular}{|l|}
\hline Sialorrea secundaria a \\
\hline Desórdenes neurológicos \\
\hline Cánceres de cabeza y cuello \\
\hline Cirugías oncológicas y reconstructivas en cabeza y cuello \\
\hline Trastornos sensitivos de laringe \\
\hline Fístulas salivales postquirúrgicas \\
\hline Sialocele \\
\hline Sialoadenitis crónica \\
\hline
\end{tabular}

estudios para definir el rol de la toxina botulínica en su manejo.

Como fue mencionado anteriormente, la sialorrea se presenta principalmente en pacientes con daño neurológico desde la edad pediátrica hasta adultos mayores, por lo que además se caracterizan por tener múltiples comorbilidades asociadas. Existe un consenso publicado el año 2010 que agrupa las principales recomendaciones en este tipo de pacientes y que recoge los resultados de varios estudios aquí presentados ${ }^{36}$.

La dosis recomendada en el consenso y que corresponde a la dosis utilizada en todos los estudios fluctúa entre $15 \mathrm{UI}$ a $50 \mathrm{UI}$ de Botox ${ }^{\circledR}$ y 15 UI a 75 UI de Dysport ${ }^{\circledR}$ en cada glándula parótida y submandibular. El procedimiento debe repetirse cada 4 a 6 meses.

Se punciona en dos a tres puntos cada glándula parótida y en uno o dos puntos la glándula submandibular. Puede realizarse con 0 sin anestesia. La mayoría de los estudios clínicos publicados realizan la punción bajo visión ecográfica lo que ayudaría a disminuir el riesgo de complicaciones principalmente durante la inyección en la glándula submandibular ${ }^{37,38}$.

Existen trabajos que han evaluado la efectividad y perfil de efectos adversos en pacientes de edad pediátrica con parálisis cerebral de distinto grado $^{39-42} \mathrm{y}$ en adultos mayores con Parkinson ${ }^{43-46}$.

Con respecto a la evidencia disponible en estos pacientes se concluye que la inyección de toxina botulínica en la glándula parótida y submandibular es efectiva en disminuir el flujo de saliva y la percepción de salivación por parte de pacientes y cuidadores. Los estudios también muestran mejoría en la calidad de vida.

Los efectos adversos reportados en la literatura varían desde el $0 \%$ hasta el $18,7 \%{ }^{47}$.

Se dividen entre aquellos derivados de la punción propiamente tal (dolor, hematoma, sangrado) y aquellos derivados de la toxina misma (debilidad muscular). Los principales reportados en los estudios son la disfagia, la dificultad para masticar y problemas respiratorios como infecciones respiratorias bajas. En los estudios revisados no se reportan muertes ni secuelas permanentes producto de la aplicación de toxina botulínica. Los efectos adversos fueron leves a moderados y transitorios, desapareciendo a partir de la segunda semana. Se observó que quienes presentaban estos efectos colaterales tenían el antecedente de haberlos presentado, previo a la inyección, producto de su patología de base. Un estudio evaluó estos efectos adversos en niños con parálisis cerebral, muy secuelados ${ }^{48}$ y concluyó que aun en este tipo de pacientes el uso de toxina botulínica es seguro, observó que todos los eventos se produjeron dentro de los primeros 14 días y no duraron más allá de los 20.

Lo más importante al momento de decidir a quién tratar es el deseo del paciente o del cuidador de resolver la sialorrea. Otro factor a tener en cuenta es el uso previo de toxina botulínica durante los 3 meses previos para evitar efectos indeseados.

Finalmente, los estudios muestran que la inyección de toxina botulínica representa una opción mínimamente invasiva, efectiva en reducir el flujo salival y segura incluso en pacientes con grado importante de deterioro neurológico.

En patología oral se ha utilizado toxina botulínica para el manejo de trastornos de la articulación temporomandibular, específicamente en el espasmo del músculo pterigodeo lateral y bruxismo.

En ambas patologías existen estudios clínicos randomizados que apoyan el uso de la toxina en estos pacientes, el principal outcome medido es la mejora en el dolor miofacial ${ }^{49,50}$.

\section{6) Patología de nariz}

Se ha probado el uso de toxina botulínica en pacientes con rinitis alérgica, vasomotora e idiopática. 
Existen al menos 6 estudios clínicos randomizados $^{51-56}$ que comparan la inyección de toxina botulínica en los cornetes (sólo inferiores, sólo medios o ambos) utilizando distintas formulaciones de toxina botulínica (hubo un estudio que en vez de inyectar los cornetes aplicó la toxina de forma tópica).

Todos concluyen que produce una mejoría sintomática con respecto a placebo y al menos un efecto comparable y duradero en relación al tratamiento de primera línea (antihistamínicos en rinitis alérgica y bromuro de ipatropio en rinitis vasomotora). Los principales efectos adversos reportados son epistaxis leve y sequedad nasal.

Dado que es un procedimiento que genera dolor al paciente y es de alto costo su uso no sería recomendable como primera línea de tratamiento pese a existir buena evidencia al respecto. Debido a esto la toxina botulínica podría tener un rol en aquellos pacientes que a pesar de estar con tratamiento médico óptimo no logran una mejoría significativa de sus síntomas.

\section{DISCUSIÓN}

Desde el descubrimiento de la toxina botulínica y su aplicación terapéutica se ha masificado su uso en múltiples patologías.

Si bien su uso estético es el más conocido, actualmente representa el tratamiento de primera línea para un gran número de patologías.

En este artículo fueron revisados aspectos generales acerca del uso de la toxina botulínica y la evidencia en una amplia gama de patología otorrinolaringológica.

En las Tablas 3 y 4 se resumen las patologías revisadas y el nivel de evidencia que actualmente posee el uso de la toxina botulínica en cada una de ellas y su significado correspondiente.

Actualmente, la TbA se considera un medicamento seguro y efectivo en manos de profesionales capacitados para utilizarla y representa una intervención mínimamente invasiva.

En general la dosis depende de cada patología e incluso varía dentro de esta misma. La recomen-

Tabla 3.

\begin{tabular}{|l|l|}
\hline Patología facial & Nivel de evidencia \\
\hline Distonía cervical & $1 \mathrm{~A}$ \\
\hline Blefaroespasmo & $1 \mathrm{~B}$ \\
\hline Espasmo hemifacial & $1 \mathrm{~B}$ \\
\hline Parálisis facial & 4 \\
\hline Neuralgia del trigémino & $2 \mathrm{~B}$ \\
\hline Patología de oído & \\
\hline Tinnitus por mioclonías & 4 \\
\hline Patología laríngea & \\
\hline Disfonía espasmódica & $1 \mathrm{~A}$ \\
\hline Temblor laríngeo & $1 \mathrm{C}$ \\
\hline Voz en pacientes laringectomizados & $2 \mathrm{C}$ \\
\hline Tics vocales y tartamudez & $2 \mathrm{~B}$ y 4 respectivamente \\
\hline Patología autonómica & \\
\hline Sindrome de Frey & 4 \\
\hline Patología oral y de glándulas salivales & \\
\hline Sialorrea & $1 \mathrm{~B}$ \\
\hline Bruxismo y patología de ATM & $1 \mathrm{~B}$ \\
\hline Patología de nariz & \\
\hline Rinitis & $1 \mathrm{~B}$ \\
\hline
\end{tabular}


Tabla 4.

\begin{tabular}{|c|l|}
\hline Niveles de evidencia & \multicolumn{1}{|c|}{ Significado } \\
\hline AA & Revisiones sistemáticas de estudios clínicos randomizados homogéneos \\
\hline B B & Ensayos clínicos randomizados con intervalo de confianza estrecho \\
\hline CC & Estudios de práctica clínica \\
\hline $2 A$ & Revisión sistemática de estudios de cohorte \\
\hline $2 B$ & Estudio de cohorte o ensayo clínico randomizado de baja calidad \\
\hline 3 A & Revisión sistemática de casos y controles \\
\hline $3 B$ & Estudio de casos y controles \\
\hline 4 & Estudio de cohorte/series de casos/casos y controles de baja calidad \\
\hline 5 & Opinión de expertos \\
\hline
\end{tabular}

dación es ajustar la dosis según la respuesta del paciente en sesiones posteriores.

Con respecto a la forma de administración existen diferentes opciones que dependerán de la experiencia del equipo, sin embargo, los estudios recomiendan que sea guiado bajo ecografía o EMG ya que permite disminuir las complicaciones sobre todo en grupos con poca experiencia.

Las complicaciones derivadas de su uso en patología otorrinolaringológica son producto de la difusión de la toxina a musculatura adyacente. No existen casos reportados de muerte y los efectos adversos reportados son leves y transitorios.

Representa la primera línea de tratamiento en disfonía espasmódica, la distonía cervical y presenta buenos resultados en temblor laríngeo.

Representa una opción segura y efectiva para el manejo de la sialorrea en pacientes neurológicos y en otras patologías como espasmo hemifacial, blefaroespasmo, bruxismo y patologías de la ATM.

En el tratamiento de la rinitis podría tener algún rol en pacientes refractarios a manejo médico óptimo.

En cuanto a las limitaciones de este artículo, no se incluyeron estudios que evaluaran la toxina botulínica $B$ dado que no está disponible en nuestro país, sin embargo se utiliza en otros países en casos refractarios a TbA.

En conclusión, la toxina botulínica es un medicamento seguro y efectivo en las patologías descritas.

\section{BIBLIOGRAFÍA}

1. Schaffner W. Clostridium botulinum. En: Mandell, Douglas \& Bennett's Principles and Practice of Infectious Diseases. Mandell GL, Bennett JE and Dolin R, editors. $3^{\text {rd }}$ edit., Churchill Livingstone, NY 1990, pp: 1847-50.

2. van Ermengem E. Ueber einen neuen anaeroben Bacillus und seine Beziehungen zum Botulismus. Zeitschrift für Hygiene und Infektionskrankheiten 1897; 26: 1-56 (translation reprinted in Rev Infect Dis 1979; 1: 701-19).

3. Walter Ledermann D. Historia del Clostridium botulinum. Rev Chil Infect Edición aniversario 2003; 39-41.

4. Carruthers J, Fournier N, Kerscher M, Ruiz-Avila J, Trindade de Almeida AR and Kaeuper G. The convergence of medicine and neurotoxins: $A$ focus on botulnum toxin type $A$ and its application in aesthetic medicine-A global, evidence based botulinum toxin consensus education initiative. Dermatologic Surgery 2013; 39: 510-25. doi: 10.1111/dsu.12148.

5. Blitzer A \& Sulica L. Botulinum toxin: basic science and clinical uses in otolaryngology. The Laryngoscope 2001; 111(2): 218-26.

6. dePaiva A, Meunier FA, Molgo J, Aoki KR, Dolly J0. Functional repair of motor endplates after botulinum neurotoxin type A poisoning: biphasic switch of synaptic activity between nerve sprouts and their parent terminals. Proc Natl Acad Sci USA 1999; 96: 3200-5. 
7. Brin MF. Botulinum toxin therapy: basic science and over- view of other therapeutic applications. In: Blitzer A, Binder WJ, Boyd JB, Carruthers A, eds. Management of Facial Lines and Wrinkles. Philadelphia: Lippincott Williams \& Wilkins, 2000; 279-302.

8. ScotT AB, SuzUKI D. Systemic toxicity of botulinum toxin by intramuscular injection in the monkey. Mov Disord 1988; 3: 333-5.

9. Greene P, Fahn S, Diamond B. Development of resistance to botulinum toxin type $A$ in patients with torticollis. Mov Disord 1994; 9: 213-7.

10. Fahn S LT, Moslowitz C, Brin M, Bressman S, Burke $R$, Scott A. Double blind controlled study of botulinum toxin for blepharospasm. Neurology 1985; 35(Suppl): 271-2.

11. Jankovic J. Blepharospasm and oromandibularlaryngeal-cervical dystonia: a controlled trial of botulinum a toxin therapy. Adv Neurol 1988; 50: 583-91.

12. Costa J, Espirito-Santo C, Borges A, et al. Botulinum toxin type a therapy for cervical dystonia. Cochrane Database Syst Rev 2005; 1 : CD003633.

13. Yoshimura DM, Aminoff MJ, Tami TA, Scott AB. Treatment of hemifacial spasm with botulinum toxin. Muscle Nerve 1992; 15: 1045-9.

14. Toffola ED, Furini F, Redaelli C, Prestifilippo E, BEJOR M. Evaluation and treatment of synkinesis with botulinum toxin following facial nerve palsy. Disabil Rehabil 2010; 32: 1414-8.

15. Reddy UP, Woodward JA. Abobotulinum toxin a (dysport) and botulinum toxin type a (botox) for purposeful induction of eyelid ptosis. Ophthal Plast Reconstr Surg 2010; 26: 489-91.

16. Turk U, Ilhan S, Alp R, Sur H. Botulinum toxin and intractable trigeminal neuralgia. Clin Neuropharmacol 2005; 28: 161-2.

17. Bohluli B, Motamedi MH, Bagheri SC, et al. Use of botulinum toxin a for drug-refractory trigeminal neuralgia: preliminary report. Oral Surg Oral Med Oral Pathol Oral Radiol Endod 2011; 111: 47-50.

18. Langevin P, Peloso PM, Lowcock J, et al. Botulinum toxin for subacute/chronic neck pain. Cochrane Database Syst Rev 2011; 7: CD008626.

19. Conill Tobias N, Paula Vernetta CD, García Callejo FJ, Marco Algarra J. Objective tinnitus from palatal myoclonus. Use of botulinum toxin: a case report. Acta Otorrinolaringol Esp 2012; 63 : 391-2.

20. LIU HB, Fan JP, LIN SZ, Zhao SW, Lin Z. Botox transient treatment of tinnitus due to stapedius myoclonus: case report. Clin Neurol Neurosurg 2011; 113: 57-8.

21. Stidham KR, Solomon PH \& Roberson JB. Evaluation of botulinum toxin $A$ in treatment of tinnitus. Otolaryngology Head and Neck Surgery 2005; 132(6): 883-9.

22. Fulmer SL, Merati AL \& Blumin JH. Efficacy of laryngeal botulinum toxin injection: Comparison of two techniques. The Laryngoscope 2011; 121(9): 1024-8.

23. Asgeirsson H, Jakobsson F, Hualtason H, Jonsdottir $\mathrm{H}$, Sveinbjornsdottir S. Prevalence study of primary dystonia in Iceland. Mov Disord 2006; 21: 293-8.

24. Blitzer A. Spasmodic dysphonia and botulinum toxin: Experience from the largest treatment series. European Journal of Neurology 2010; 17(s1): 28-30.

25. Adler CH, Bansberg SF, Hentz JG, Ramig LO, Buder EH, WITT K \& CAVINESS JN. Botulinum toxin type A for treating voice tremor. Archives of Neurology 2004; 61(9): 1416-20.

26. Warrick P, Dromey C, Irish JC, Durkin L, Pakian A \& Lang A. Botulinum toxin for essential tremor of the voice with multiple anatomical sites of tremor: A crossover design study of unilateral versus bilateral injection. The Laryngoscope 2000; 110(8): 1366-74.

27. Barkmeier-Kraemer J, Lato A \& Wiley K. Development of a speech treatment program for a client with essential vocal tremor. In: Seminars in speech and language 2011; 32(1): 43.

28. Lewin JS, Bishop-Leone JK, Forman AD, Díaz EM JR. Further experience with botox injection for tracheoesophageal speech failure. Head Neck 2001; 23: 456-60.

29. Meleca RJ, Dworkin JP, Zormeier MM, Simpson ML, Shibuya T, Mathog RH. Videostroboscopy of the pharyngoesophageal segment in laryngectomy patients treated with botulinum toxin. Otolaryngol Head Neck Surg 2000; 123: 38-43.

30. Zormeier MM, Meleca RJ, Simpson ML, et al. Botulinum toxin injection to improve tracheoesophageal speech after total laryngectomy. Otolaryngol Head Neck Surg 1999; 120: 314-9. 
31. Bartolomel L, Marsala SZ, Pighi GP, Cristofori V, Pagano G, Pontarin M \& Marchin C. Botulinum toxin type $A$ : An effective treatment to restore phonation in laryngectomized patients unable to voice. Neurological Sciences 2011; 32(3): 443-7.

32. WITT RL \& PRIBITKIN EA. How can Frey's syndrome be prevented or treated following parotid surgery? The Laryngoscope 2013; 123(7): 1573-4.

33. de Bree R, Duyndam Je, Kuik DJ, Leemans CR. Repeated botulinum toxin type a injections to treat patients with Frey syndrome. Arch Otolaryngol Head Neck Surg 2009; 135: 287-90.

34. Ellies M, Laskawi R, Rohrbach-Volland S, Arglebe $C \&$ BEUCHE W. Botulinum toxin to reduce saliva flow: Selected indications for ultrasound-guided toxin application into salivary glands. The Laryngoscope 2002; 112(1): 82-6.

35. Ellies M, Gottstein U, Rohrbach-Volland S, Arglebe C, LASKAWI R. Reduction of salivary flow with botulinum toxin: extended report on 33 patients with drooling, salivary fistulas, and sialadentis. Laryngoscope 2004; 114: 1856-60.

36. Redihough D, Erasmus CE, Johnson H, McKellar GMW \& Jongerius PH. Botulinum toxin assessment, intervention and aftercare for paediatric and adult drooling: International consensus statement. European Journal of Neurology 2010; 17(s2): 109-21.

37. Dogu 0, Apaydin D, Sevim S, Talas DU, Aral M. Ultrasound-guided versus "blind" intraparotid injections of botulinum toxin-A for the treatment of sialorrhoea in patients with ParkinsonÕs disease. Clin Neurol Neurosurg 2004; 106: 93-6.

38. Jongerius PH, Joosten F, Hoogen FJ, Gabreels FJ, Rotteveel JJ. The treatment of drooling by ultrasound- guided intraglandular injections of botulinum toxin type A into the salivary glands. Laryngoscope 2003; 113: 107-11.

39. Jongerius PH, Rotteveel JJ, van Limbeek J, Gabree Ls FJ, van Hulst K, van den Hoogen FJ. Botulinum toxin effect on salivary flow rate in children with cerebral palsy. Neurology 2004; 63: 1371-5.

40. ONG LC, Wong SW, Hamid HA. Treatment of drooling in children with cerebral palsy using ultrasound guided intraglandular injections of botulinum toxin A. J Pediatr Neurol 2009; 7: 141-5.

41. Banerjee KJ, Glasson C, OÕFlaherty SJ. Parotid and submandibular botulinum toxin A injections for sialorrhoea in children with cerebral palsy. Dev Med Child Neurol 2006; 48: 883-7.

42. Savarese R, Diamond M, Elovic E, Millis SR. Intraparotid injection of botulinum toxin $A$ as a treatment to control sialorrhea in children with cerebral palsy. Am J Phys Med Rehabil 2004; 83: 304-11.

43. Lagalla G, Millevolte M, Capecci M, Provinciali L, Ceravolo MG. Botulinum toxin type $A$ for drooling in Parkinson's disease: a double-blind, randomized, placebo- controlled study. Mov Disord 2006; 21: 704-7.

44. Mancini F, Zangaglia R, Cristina S, et al. Doubleblind, placebo-controlled study to evaluate the efficacy and safety of botulinum toxin type A in the treatment of drooling in Parkinsonism. Mov Disord 2003; 18: 685-8.

45. Friedman A, Potulska A. Quantitative assessment of parkinsonian sialorrhea and results of treatment with botulinum toxin. Parkinsonism Relat Disord 2001; 7: 329-32.

46. Su CS, LaN MY, LIU JS, ET AL. Botulinum toxin type A treatment for Parkinsonian patients with moderate to severe sialorrhea. Acta Neurol Taiwan 2006; 15: 170-6.

47.Khan WU, Campisi P, Nadarajah S, et al. Botulinum toxin A for treatment of sialorrhea in children: an effective, minimally invasive approach. Arch Otolaryngol Head Neck Surg 2011; 137(4): 339-44.

48. O'Flaherty SJ, Janakan V, Morrow AM, Scheinberg AM \& WAUGH MCA. Adverse events and health status following botulinum toxin type A injections in children with cerebral palsy. Developmental Medicine \& Child Neurology 2011; 53(2): 12530.

49. Denglehem C, Maes JM, Raoul G, Ferri J. [Botulinum toxin a: analgesic treatment for temporomandibular joint disorders]. Rev Stomatol Chir Maxillofac 2012; 113: 27-31.

50. Guarda-Nardini L, Manfredini D, Salamone M, Salmaso L, Tonello S, Ferronato G. Efficacy of botulinum toxin in treating myofascial pain in bruxers: a controlled placebo pilot study. Cranio 2008; 26: 126-35. 
51. YANG TY, JUNG YG, KIM YH, JANG TY. A comparison of the effects of botulinum toxin a and steroid injection on nasal allergy. Otolaryngol Head Neck Surg 2008; 139: 367-71.

52. Rohrbach S, Junghans K, Kohler S, Laskawi R. Minimally invasive application of botulinum toxin a in patients with idiopathic rhinitis. Head Face Med 2009; 5: 18.

53. SaPCI T, YaZICI S, EvcImIK MF, et al. Investigation of the effects of intranasal botulinum toxin type $a$ and ipratropium bromide nasal spray on nasal hypersecretion in idiopathic rhinitis without eosinophilia. Rhinology 2008; 46: 45-51-

54. Ozcan C, Vayisoglu Y, Dogu 0, Gorur K. The effect of intranasal injection of botulinum toxin a on the symptoms of vasomotor rhinitis. $A m \mathrm{~J}$ Otolaryngol 2006; 27: 314-8.

55. Nowak K, Szyfter W. [Application of botulinum toxin a in chronic intrinsic rhinitis]. Otolaryngol Pol 2011; 65: 103-5.

56. Hashemi SM, Okhovat A, Aminı $S$ \& Pourghasemian $M$. Comparing the effects of botulinum toxin $A$ and cetirizine on the treatment of allergic rhinitis. Allergology International 2013; 62: 245-9. 